\title{
Magnetic stent hyperthermia for esophageal cancer: An in vitro investigation in the ECA-109 cell line
}

\author{
JIA-YI LIU ${ }^{1,2^{*}}$, LING-YUN ZHAO $^{2 *}$, YU-YING WANG ${ }^{2,3}$, DAN-YE LI ${ }^{1,2}$, \\ DAN TAO ${ }^{4}$, LI-YA LI $^{4}$ and JIN-TIAN TANG ${ }^{1,2}$ \\ ${ }^{1}$ Department of Oncology, Xiangya Hospital, Central South University, Changsha, Hunan Province 410008; ${ }^{2}$ Department of \\ Engineering Physics, Institute of Medical Physics and Engineering, Key Laboratory of Particle and Radiation Imaging, \\ Ministry of Education, Tsinghua University, Beijing 100084; ${ }^{3}$ Department of Bio-Pharmaceutics, Beijing University \\ of Chinese Medicine, Beijing 100102; ${ }^{4}$ China-Japan Friendship Hospital, Beijing 100029, P.R. China
}

Received August 1, 2011; Accepted September 15, 2011

DOI: 10.3892/or.2011.1603

\begin{abstract}
Magnetic stent hyperthermia (MSH) is a novel approach for targeted thermotherapy for esophageal cancer, which is based on the mechanism that inductive heat can be generated by the esophageal stent upon exposure under an alternative magnetic field (AMF). A positive effect of MSH on esophageal cancer has been demonstrated, however, there is no study on the in vitro effects of heating treatment or of the effects of AMF exposure on human esophageal cancer cells. This study aimed to investigate the effect of MSH and of AMF exposure in esophageal cancer cells. Inductive heating characteristics of esophageal stents were assessed by exposing the stents under AMF. A rather rapid temperature rise of the Ni-Ti stent when subjected to AMF exposure was observed and the desired hyperthermic temperature could be controlled by adjusting the field parameter of the AMF. Human esophageal squamous carcinoma (ESCC) ECA-109 cells were divided into four groups: the control group, the water-bath heating group, the MSH group and the AMF exposure group. Hyperthermic temperatures were 43,48 and $53^{\circ} \mathrm{C}$ and the treatment time was in the range of 5-30 min. The MTT assay, apoptotic analysis and TUNEL staining were applied in the current investigation. Exposure of ECA-109 cells under AMF with a field intensity of 50 to $110 \mathrm{kA} / \mathrm{m}$ had negligible effect on cell viability, cell necrosis and apoptosis. Hyperthermia had a remarkable inhibitory effect on the cell viability and the effect was dependent on
\end{abstract}

Correspondence to: Dr Jin-Tian Tang or Dr Ling-Yun Zhao, Department of Engineering Physics, Tsinghua University, Beijing 100084, P.R. China

E-mail: tangjt@tsinghua.edu.cn

E-mail: lyzhao@tsinghua.edu.cn

*Contributed equally

Key words: magnetic stent hyperthermia, esophageal cancer, thermal dose, alternative magnetic field the thermal dose (temperature and time). The optimal thermal dose of MSH for ECA-109 cells was $48^{\circ} \mathrm{C}$ for $20-30 \mathrm{~min}$. The study also elucidated that there was a difference in the effects on cell necrosis and apoptosis between the heating mode of water bath and MSH. The data suggest that MSH may have clinical significance for esophageal cancer treatment.

\section{Introduction}

Esophageal cancer constitutes one of the major causes of cancer-related death worldwide, with $\sim 462,000$ newly occurring cases and 38,600 deaths/year. It is a highly aggressive malignancy with a propensity for invasive local growth, early lymphatic spread and vascular invasion. Radical surgery as the treatment of choice offers 5-year survival rates of only $30 \%$ $(1,2)$. Therefore, an innovative medical treatment strategy for esophageal cancer is required.

It is believed that hyperthermia is a well-established rationale and promising approach for cancer therapy, as it has been proven that sustained heating treatment above certain temperature will cause cell necrosis and/or apoptosis. Moreover, as a physical treatment, hyperthermia could result in fewer side effects than chemotherapy or radiotherapy. However, the inevitable technical problem with hyperthermia is the difficulty in heating only the local tumor region to the intended temperature without damaging the surrounding healthy tissue. Targeted magnetic induction hyperthermia is expected to be a new breakthrough in tumor hyperthermia $(3,4)$. For the purpose of targeted therapy by magnetic-mediated hyperthermia (MMH), the tumor can be placed with magnetic agents, for instance, ferromagnetic alloy thermoseeds of rod shape or magnetic nanoparticles (5-8). Upon exposure to an external alternative magnetic field (AMF), inductive heat will be generated by the magnetic agents to form a high temperature zone (around $50^{\circ} \mathrm{C}$ ) at the tumor foci. Studies in the US, Germany, Japan as well as China have made significant progress in $\mathrm{MMH}$ and have led to clinical trials (9).

In recognition of the working mechanism of $\mathrm{MMH}$, which involves heating generation mediated by ferromagnetic agents under AMF exposure, it thus would be an effective and wise 

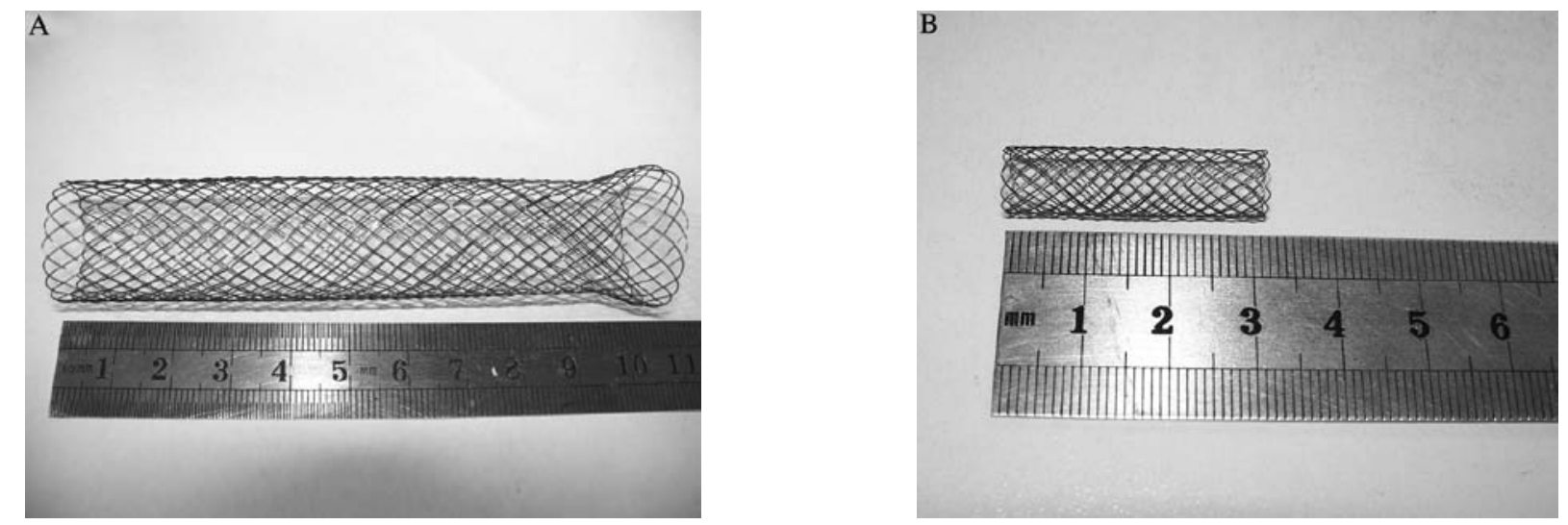

Figure 1. The two kinds of nitinol stents applied in the study. Left, the commercially available stent (type I); right, the stent for cell experiment (type II).

strategy to combine MMH with stent placement for patients with inoperable esophageal cancer. Endoscopic placement of a metal stent is already an established palliative approach for patients with advanced inoperable esophageal cancer. However, stent placement has no therapeutic effect for esophageal cancer treatment. This hyperthermia treatment combined with medical stent placement, which can be termed as magnetic stent hyperthermia (MSH), can be regarded as one category of MMH. Though much research has been carried out on MMH by applying magnetic nanoparticles or thermalseed as the hyperthermia agent, little attention has been paid to MSH. Yambe et al (10) performed animal experiments by using goats with the same weight as the average Japanese patient and they found that the hyperthermia effect was easily obtained by electromagnetic induction from outside the goat's body. Meanwhile, Akiyama et al carried out some pioneering clinical work on the feasibility and effectiveness of MSH. MSH was combined with simultaneous chemotherapy in 13 patients and radiochemotherapy in 5 patients (11). Their results indicated that MSH proved effective in 8 of 9 patients who received the treatment at least three times. The clinical results suggest that MSH can improve the effectiveness of combination therapy and suppress local tumor growth. Our research group also initiated some investigation for esophageal treatment. We successfully set up the tumor model for esophageal cancer on New Zealand white rabbits and we found that it was safe to perform MSH for 10 min on the rabbit normal esophageal wall and that tumor growth was delayed (12).

A positive effect of MSH on esophageal cancer has been demonstrated and a clinical investigation has suggested that the addition of local hyperthermia to radio-chemotherapy was effective for patients, however, the effects of heating treatment or of AMF exposure in esophageal cells have not been evaluated. Herein, a systematic investigation was carried out to study the effects of heating treatment and AMF exposure in human esophageal cancer cells. Both the traditional water bath heating treatment as well as MSH treatment were employed to compare the effect of the heating mode on the esophageal cancer cells. ECA-109, an esophageal squamous carcinoma (ESCC) cell line was used. The choice of ESCC is based on the fact that although the incidence of esophageal adenocarcinoma is rising in Western countries, esophageal squamous cell carcinoma remains the dominant type in Asia
(13). Our results will provide useful information for the understanding of the mechanism of heating treatment and AMF exposure in human esophageal cancer cells. The deductions from the experiments may have clinical significance for the use of MSH in esophageal cancer treatment.

\section{Materials and methods}

Cell culture and reagents. In the present study, the ESCC cell line ECA-109 was provided by Cell Center, Institute of Basic Medical Sciences, Chinese Academy of Medical Sciences and Peking Union Medical College. The cells were cultured in Dulbecco's modified Eagle's medium (DMEM) supplemented with $4.5 \mathrm{~g} / 1$ glucose, $1.5 \mathrm{~g} / 1$ sodium bicarbonate, $100 \mathrm{U} / \mathrm{ml}$ penicillin, $100 \mathrm{mg} / \mathrm{ml}$ streptomycin, and $10 \%$ fetal calf serum (FCS) under standard conditions (humidified atmosphere of $95 \%$ air plus $5 \% \mathrm{CO}_{2}, 37^{\circ} \mathrm{C}$ ).

3-(4,5-Dimethylthiazol-2-yl)-3,5-diphenyl tetrazolium bromide (MTT) was provided by Biodee, Biotechnology Co., Ltd. (Beijing, China). The Annexin V-FITC kit was purchased from Kaiji Co., Ltd. (Nanjing, China) and used as described by the provider. Solvents with analytical grade were obtained from commercial suppliers and used without further purification unless otherwise indicated.

Esophageal stent, applicator for AMF and temperature measurement. Two types of superelastic nitinol stents provided by the Beijing Institute of Nonferrous Metals, (Beijing, China) were applied in the current study. Both stents were made of nickel-titanium (Ni-Ti) alloy wire with a wire diameter of $0.16 \mathrm{~mm}$, but were of different size. A commercially available esophageal stent in clinical application with a length of $110 \mathrm{~mm}$ and a macroscopic diameter of $15 \mathrm{~mm}$ (type I) was used to study the heating inductive property of the stent upon exposure under AMF. For the cell experiment, the size of the stent was specially designed with the length of $42 \mathrm{~mm}$ and a macroscopic diameter of $8 \mathrm{~mm}$ (type II). The two types of stents are shown in Fig. 1.

The whole-body applicator of AMF for clinical application was developed and designed by our research group and was manufactured by Shuangping Instrument Technology, Co. Ltd, Shenzhen, Guangzhou Province, China. The AMF applicator was $110 \mathrm{kHz}$ with an adjustable field intensity of 15 to $50 \mathrm{kA} / \mathrm{m}$ 


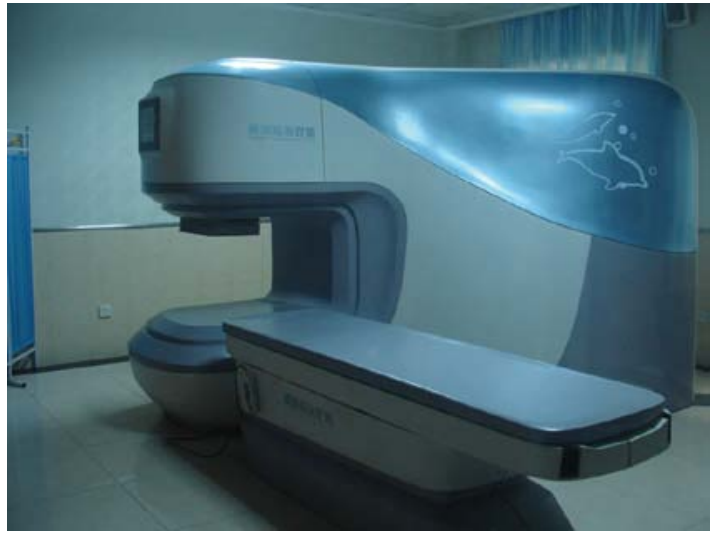

Figure 2. Whole-body applicator of AMF in a clinical trial.

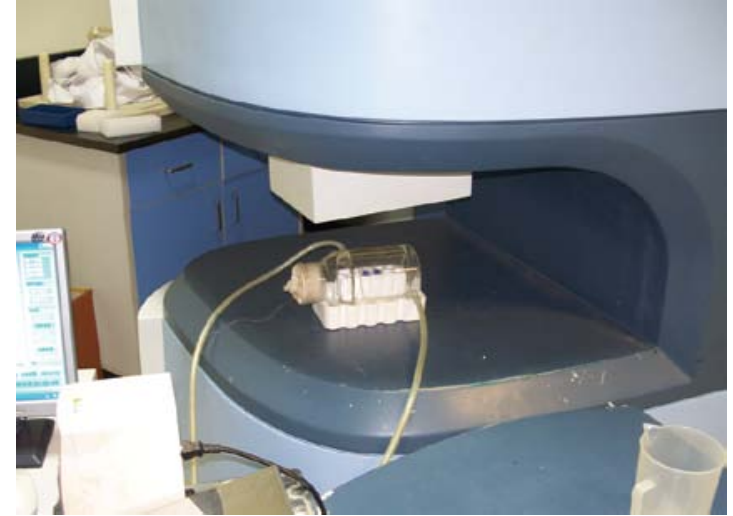

Figure 3. Experimental apparatus used to investigate the inductive heating profile of the esophageal stent under the AMF.

apoptosis were conducted after the cells were recovered under standard culture conditions at $37^{\circ} \mathrm{C}$ in $5 \% \mathrm{CO}_{2}$. cator passed the qualification test on Evaluation of Biology of Medical Apparatus GB/T 16886.15/ISO 10993-15 and approved by the State Food and Drug Administration (SFDA) for clinical trials. The appearance of the applicator is shown in Fig. 2.

A thermal-couple temperature probe (Model IT-18, Copper-Constantan, Physitemp, Clifton, NJ, USA) was applied for the temperature measurement. The probe fibers were connected to a 4-channel millivoltmeter (Model XSOL-4, Beijing Kunlun Tianchen Instrument Technology, Co., Ltd., Beijing, China) and the data were collected every $12 \mathrm{sec}$ by PC with home-written software.

Method of heating. A water-jacket incubator was designed for temperature maintenance. The jacket was connected with a water bath so that the temperature inside the jacket could be adjusted and maintained around $37^{\circ} \mathrm{C}$ throughout the study. For the purpose of testing the temperature profile of the stents, the stents of type I were placed inside the jacket before they were subjected to the AMF as shown in Fig. 3.

ECA-109 cells in the exponential phase were subjected to heating treatment. The harvesting cells were transferred to cryogenic vials and cells were divided into four groups: the negative control group in which cells were routinely cultured (group A); the water bath heating group (group B); the MSH group (group C); and the AMF exposure group (group D). Two modes of heating treatment were applied in the current investigation. For the water bath heating (group B), the cell-loaded cryogenic vials were incubated in a hot water bath at temperatures of $37,43,48$ and $53^{\circ} \mathrm{C}$ for different treatment times. Under the mode of MSH (group C), the Ni-Ti stent (type II) was put inside the cell-loaded cryogenic vial. The vial was then placed inside the water-jacket incubator as mentioned above. Upon heating treatment, the field intensity of the AMF was adjusted to obtain the desired hyperthermic temperature for cell heating treatment. For both heating mode, the temperature inside the cryogenic vial was monitored by the thermocouple. In order to elucidate the effect of AMF exposure on the proliferation and apoptosis of ECA-109 cells, a positive control group (group D) was set for cell AMF exposure only. The cell-loaded cryogenic vial without a stent inside was exposed under the same condition as the cells subjected to MSH. After each treatment, further investigations on cell viability and cell
Cell viability analysis by the MTT assay. Cell viability of ECA-109 cells after each treatment was determined by the MTT assay. Briefly, $5 \times 10^{3}$ cells/well were seeded in 96 -well plates. After incubation for $4 \mathrm{~h}, 20 \mu \mathrm{l}$ MTT was added to each followed by the addition of $150 \mathrm{ml}$ DMSO. After $20 \mathrm{~min}$, the amount of formazan present was quantified by determining the absorbance at $490 \mathrm{~nm}$. Results were expressed as the percentage of optical density in relation to the negative control, corresponding to the mean of three experiments.

Annexin V-FITC/PI double staining assays of the apoptotic cells. The occurrence of apoptosis and/or necrosis was evaluated by Annexin V binding and PI uptake. Positioning of quadrants on Annexin V/PI dot plots was performed and living cells (Annexin V-PI-), early apoptotic/primary apoptotic cells (Annexin $\mathrm{V}^{+} / \mathrm{PI}^{-}$), late apoptotic/secondary apoptotic cells (Annexin $\mathrm{V}^{+} / \mathrm{PI}^{+}$) and necrotic cells (Annexin $\mathrm{V}^{-} / \mathrm{PI}^{+}$) were distinguished. The total apoptotic proportion included the percentage of cells that were Annexin $\mathrm{V}^{+} / \mathrm{PI}^{-}$and Annexin $\mathrm{V}^{+} / \mathrm{PI}^{+}$. Annexin-V binding was performed using an Annexin V-FITC kit as described by the manufacturer. Cells for $24 \mathrm{~h}$. The cells were pretreated with various concentrations of the methanol extract (25 and $50 \mu \mathrm{g} / \mathrm{ml})$. After $24 \mathrm{~h}$, dRib $(50 \mathrm{mM})$ was added to the plate and incubated at $37^{\circ} \mathrm{C}$ for an additional $24 \mathrm{~h}$. Finally, the cells were harvested and washed with PBS and suspended in $100 \mu \mathrm{l}$ Annexin $\mathrm{V}$ binding buffer (containing $10 \mathrm{mM}$ HEPES/NaOH, pH 7.4, $140 \mathrm{mM} \mathrm{NaCl}$, $2.5 \mathrm{mM} \mathrm{CaCl}_{2}$ ). Then, the cells were double-stained with $10 \mu \mathrm{l}$ FITC-labeled Annexin V and $10 \mu \mathrm{l}$ PI solution (containing $50 \mu \mathrm{g} / \mathrm{ml}$ in PBS). The samples were incubated for $20 \mathrm{~min}$ at room temperature and then analyzed by flow cytometry

TUNEL staining. Cover glass was cut into pieces whose shape was consistent with the size of the cryogenic vials. After cells were grown to $90 \%$ confluence on the small piece of cover glass, the cover glass was transferred to cryogenic vials and subjected to the experimental conditions. The cells were well, and the cells were incubated for an additional $4 \mathrm{~h}$ at $37^{\circ} \mathrm{C}$, were plated at a density of $1 \times 10^{6}$ cells/well into 24 -well plates performed with a FACSCalibur (Becton-Dickinson, USA). 


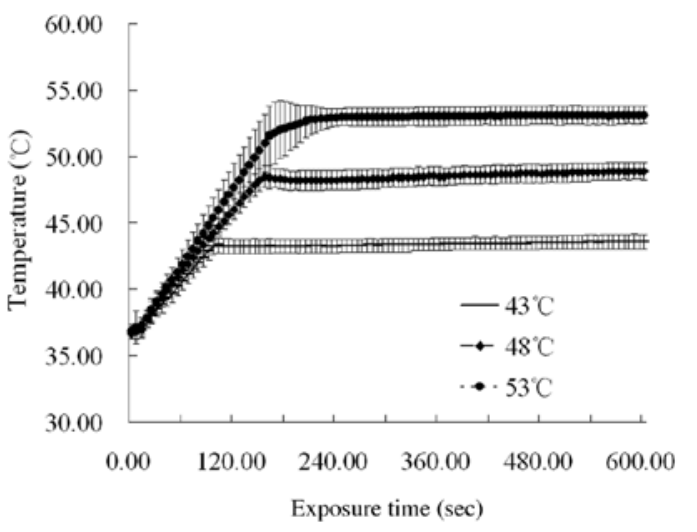

Figure 4. Inductive heating profiles of the esophageal stent under AMF. Conditions for AMF are properly controlled at the targeting temperatures of 43,48 and $53^{\circ} \mathrm{C}$.

cultured for $24 \mathrm{~h}$ and then removed from the culture medium and air dried before fixation in complete medium containing $4 \%$ paraformaldehyde for $30 \mathrm{~min}$. Cells were then washed with PBS thrice and treated with $3 \% \mathrm{H}_{2} \mathrm{O}_{2}$ in PBS to suppress endogenous peroxidase activity for $15 \mathrm{~min}$. Next, the cells were incubated in terminal deoxynucleotidyl transferase solution for $60 \mathrm{~min}$ at $37^{\circ} \mathrm{C}$. Biotin-conjugated deoxyuridine triphosphate, a transferase enzyme that attaches to the free ends of any fragmented DNA, was included in the solution and then washed in buffer and incubated in horseradish peroxidase-conjugated anti-fluorescein antibody for $30 \mathrm{~min}$ at $37^{\circ} \mathrm{C}$. After rinsing in PBS, the peroxidase was visualized by incubation with $0.1 \%$ 3,3'-diaminobenzidine and $0.3 \% \mathrm{H}_{2} \mathrm{O}_{2}$ in PBS for $10 \mathrm{~min}$.

Statistical analysis. Data are expressed as mean \pm SD. Statistical comparisons were performed by the Student's t-test or ANOVA followed by a Student Newman-Keuls multiple comparison test. A value of $\mathrm{P}<0.05$ was considered significant.

\section{Results}

Inductive heating profile of the esophageal stent. The heating profiles of the medical $\mathrm{Ni}$-Ti esophageal stent under AMF of $110 \mathrm{kHz}$ with different field intensities are shown in Fig. 4. It was noticed that regardless of the field intensity, the Ni-Ti stent possessed excellent inductive heating characteristics under AMF. Upon exposure to the AMF, rapid temperature rising as indicated by the initial slope of the curves could be observed. Fig. 4 demonstrates that the heating curve exhibits an asymptote, which signals the equilibrium of the heating process under different field intensities. The curves show that the final temperatures upon equilibrium $\left(\mathrm{T}_{\mathrm{e}}\right)$ depend strongly on the field intensity, i.e. the higher field intensity of the AMF can ensure a higher $T_{e}$, which means more energy can be generated by the stent.

We also noticed that during the process of temperature rising, the inductive heating profile of the stent under AMF can be rather reproducible under the same parameters, and the $\mathrm{T}_{\mathrm{e}}$ of each curve can be stably maintained around the desired hyperthermia temperature, for instance in our case, 43,48 and $53^{\circ} \mathrm{C}$, respectively. This observation implies that during the treatment process, the desired treatment temperature can be
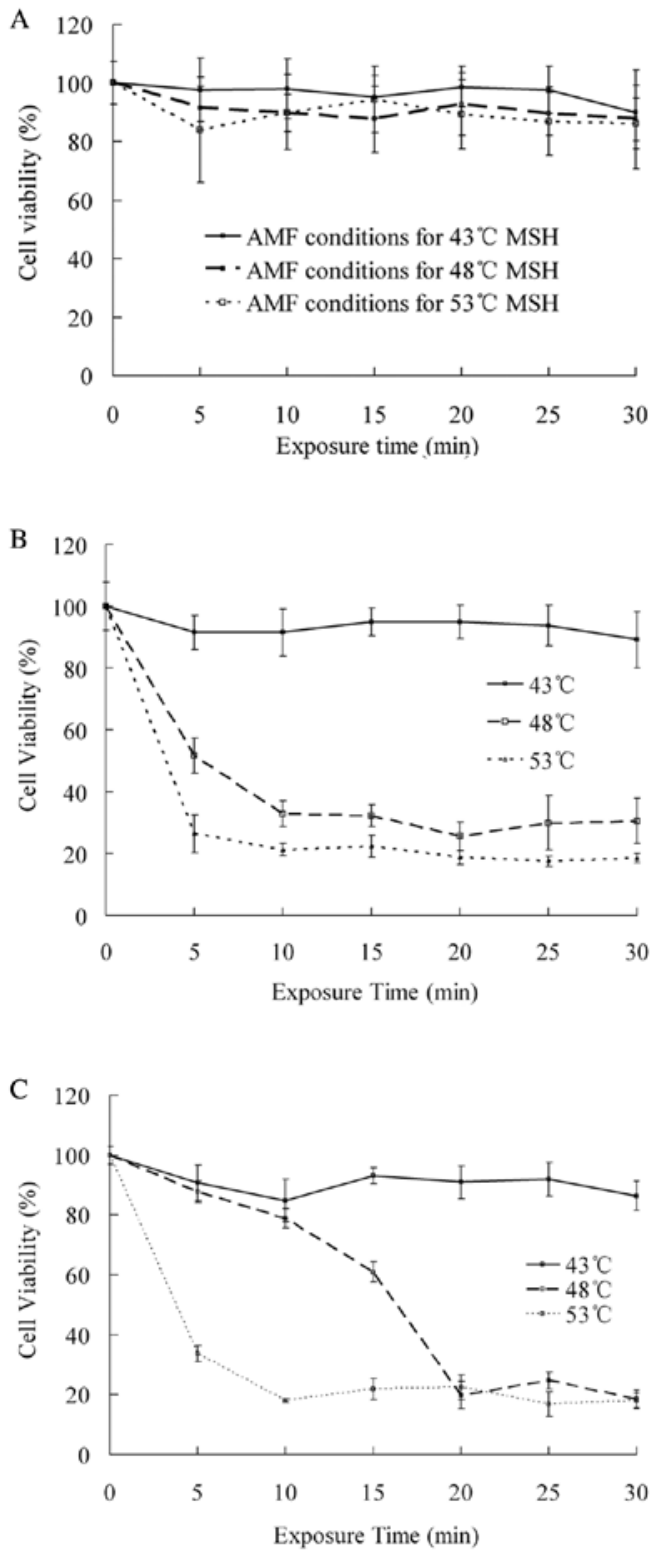

Figure 5. Effect of heating treatment and AMF exposure on ECA-109 cell viability. (A) Effect of AMF exposure; (B) water bath heating mode; (C) MSH heating mode.

conveniently controlled by adjusting the field parameter of the AMF. Fig. 4 also illustrates that the rate of temperature rising, as reflected by the slope of the temperature profile, became faster at higher field intensity. Such kind of process reproducibility, stable temperature maintenance and rapid temperature rising is very significant for cancer hyperthermia, as will be further discussed.

Inhibitory effect of hyperthermia on esophageal cancer cell proliferation. The viability of ECA-109 cells subjected to different treatments was examined. Exposure under the AMF had negligible effects on the cell proliferation, and this effect was independent of both the field intensity and exposure time. During the investigation, within the field intensity scope of $50-110 \mathrm{kA} / \mathrm{m}$, cell viability remained fairly constant regardless of the exposure time. This suggests that during the process of $\mathrm{MMH}, \mathrm{AMF}$ has little contribution to the cell proliferation. 

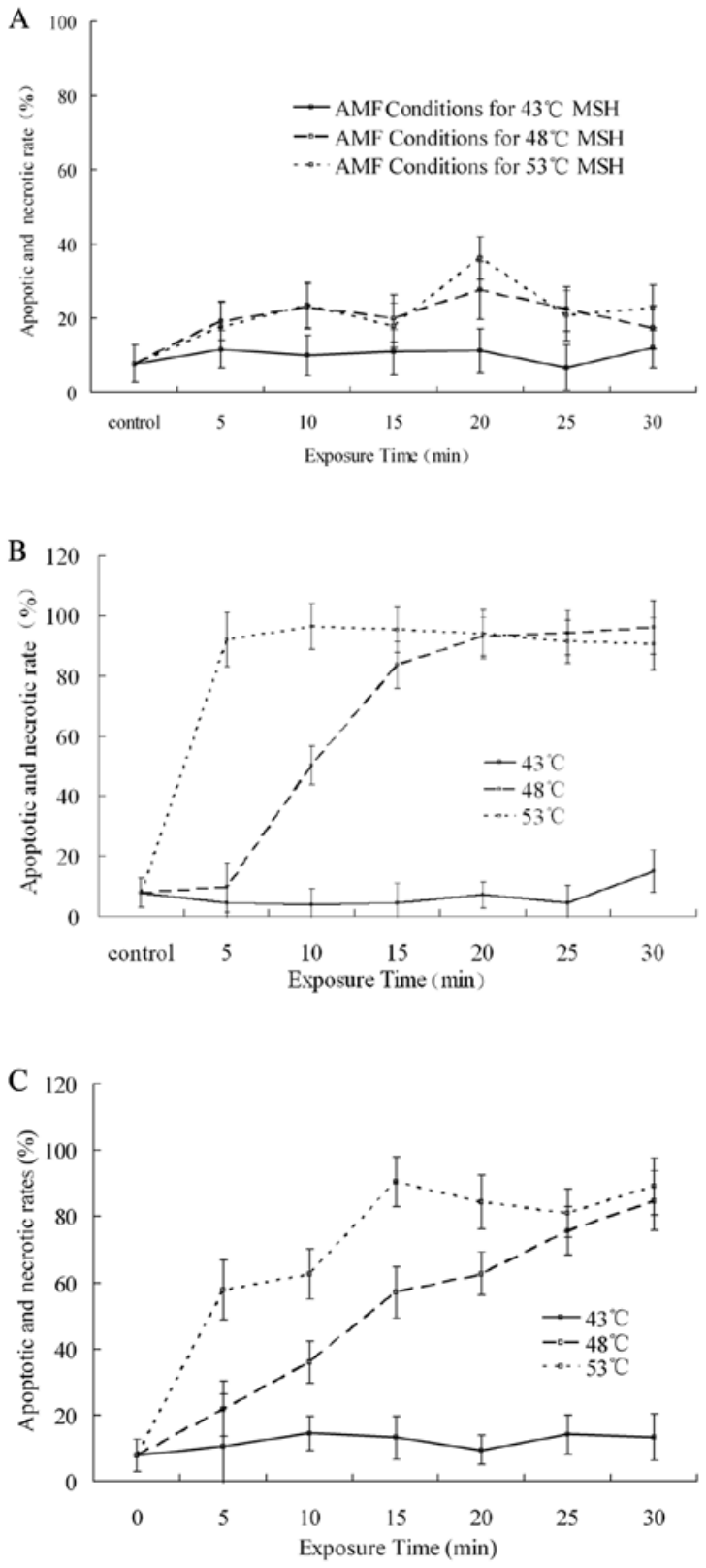

Figure 6. Time course of the effect of heating treatment and AMF exposure on ECA-109 cell apoptosis and necrosis. (A) Effect of AMF exposure; (B) water bath heating mode; (C) MSH heating mode.

Hyperthermia has a remarkable inhibitory effect on the cell viability, an effect that is temperature- and time-dependent. In terms of the temperature dependence, the higher the temperature the lower the cell viability (Fig. 5B and C). Such a phenomenon is quite easy to understand. It is also worth noting that under both modes of heating treatment, a mild temperature of $43^{\circ} \mathrm{C}$ has very little effect on the viability of ECA-109 cell in the treatment range of 5-30 min. For higher temperatures, in our case, 48 and $53^{\circ} \mathrm{C}$, the cell viability curve also demonstrates an asymptote, in which the maximal inhibitory effect at a certain temperature can be noticed. For instance, under the mode of water bath heating, cell viability was maintained at $\sim 40 \%$ after 10 min heating treatment. The viability was not further decreased even though the treatment time was prolonged to $30 \mathrm{~min}$.

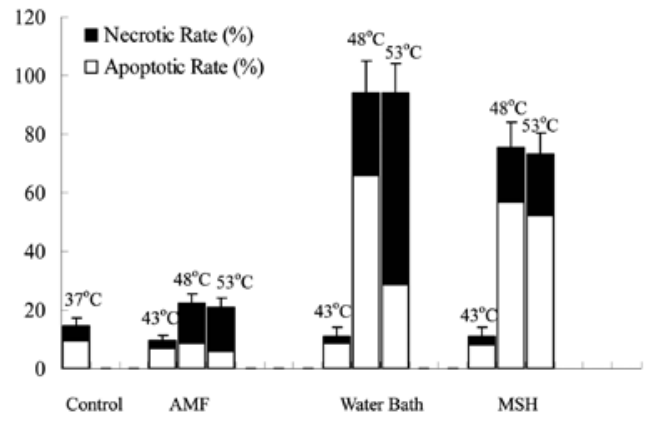

Figure 7. Effect of heating treatment and AMF exposure on ECA-109 cell apoptosis and necrosis (exposure time, $25 \mathrm{~min}$ ).

MSH can have a comparable effect as water bath hyperthermia on the viability of ECA-109 cells as demonstrated in Fig. 5B and C. Possible reason for the difference between the curves of the two figures may be the different heating transfer mechanism of the two heating treatment. Hyperthermia treatment for ECA-109 cells under $48^{\circ} \mathrm{C}$ for 20-30 min can incur a similar inhibitory effect on cell viability, as compared with that of $53^{\circ} \mathrm{C}$ heating treatment with an exposure time longer than 10 min (Fig. 5C).

Apoptosis analysis by flow cytometry. Annexin V-FITC/ PI double staining assays were performed for cell apoptosis/ necrosis analysis. As shown in Fig. 6A, compared with the cells under normal cultural condition at $37^{\circ} \mathrm{C}$, in which no significant apoptotic and necrotic cells were observed, heat treatment at $48^{\circ} \mathrm{C}$ for 25 min resulted in increased numbers of apoptotic and necrotic cells. Once again, both AMF exposure and hyperthermia at $43^{\circ} \mathrm{C}$ caused a negligible effect on cell necrosis and apoptosis, which is in good accordance with our previous observation by MTT analysis.

Apoptotic and necrotic rates of ECA-109 cells under different treatment conditions are illustrated in Fig. 7. For water bath hyperthermia, apoptosis accounts for the main reason for cell death when the cells are subjected to treatment at 43 and $48^{\circ} \mathrm{C}$, whereas treatment at $53^{\circ} \mathrm{C}$ caused the most significant enhancement of necrosis $(65.7 \%)$ rather than apoptosis $(28.6 \%)$. However, for MSH, necrosis is the dominant factor for cell death in the current investigation, regardless of the heating temperature. This phenomenon suggests that the heating mode may result in differences in cell necrosis and apoptosis rates, which differs from the results of MTT assay in which the two treating modes gave comparable results.

TUNEL assay. Findings from the TUNEL assay also support our previous phenomena from the MTT assay and flow cytometry analysis. Fig. 8 shows the TUNEL labeling of ECA-109 cells under different treatments. As compared with the staining of the cells in control group by normal culture, AMF exposure as well as $43^{\circ} \mathrm{C}$ hyperthermia for $30 \mathrm{~min}$ did not result in increased numbers of TUNEL-positive cells. However, the number of TUNEL-stained cells was remarkably increased upon cell heating by higher temperature. It is also noticed that the cell number decreased at higher temperatures and this is probably due to that cells would detach from the glass coverslide at higher temperatures. 


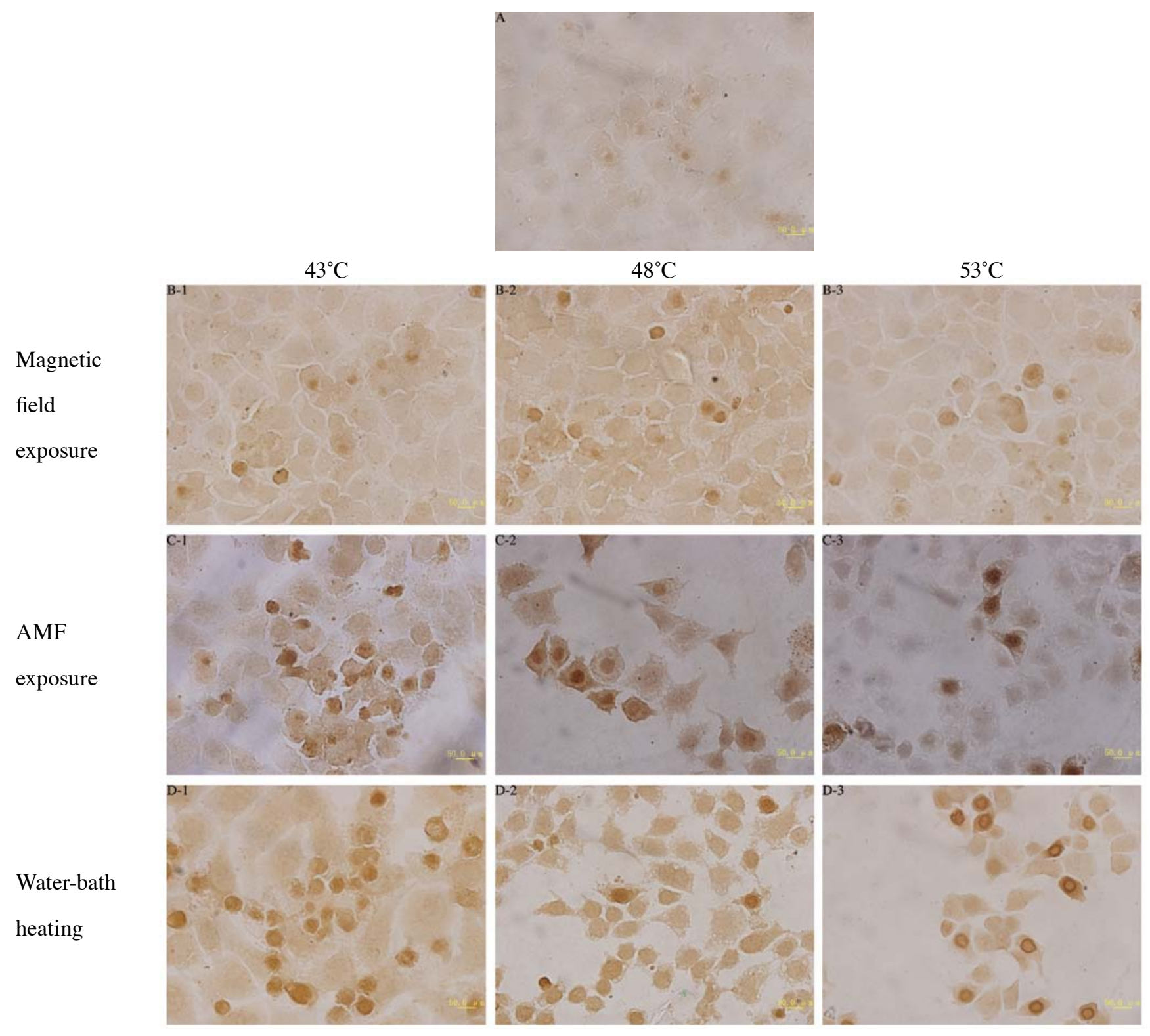

Figure 8. TUNEL labeling of ECA-109 cells under different treatments (treatment time, $25 \mathrm{~min}$ ).

\section{Discussion}

Clinically, endoscopic placement of a metal stent has become an established palliative method for advanced inoperable cases of esophageal cancer. Although stent placement is an effective palliative method for patients for the purpose to improve their nutritional status and their overall quality of life, stents can only treat obstructions mechanically for the relief of symptoms. The novel approach of MSH brings new advantages for stent placement. Except for dysphagia relief, the stent can function as a hot source upon exposure under the AMF so as to inhibit tumor growth by local heating.

In this study, our results have demonstrated that the Ni-Ti alloy stent in clinical application possesses ideal inductive heating property under the AMF. The stents can rapidly reach the desired hyperthermia temperature and the temperature can be maintained quite stably under proper AMF parameters. It should be noted that the rapid temperature rising is significantly favorable for cancer treatment by hyperthermia due to the consideration of thermotolerance. Thermotolerance indicates a temporary heat resistance following a prior heat treatment (14). If the temperature rises in a slow way within the tumor site, more thermotolerance will be induced, therefore reducing the treatment efficiency. Our study demonstrated the temperatures of the stent would get to equilibrium within $200 \mathrm{sec}$ and this can guarantee the temperature requirement for the hyperthermia for cancer treatment by avoiding thermotolerance in the meanwhile. Therefore, in the future clinical trial, there is no need to especially develop esophageal stents for MSH, which greatly facilitate the clinical application of this novel local heating treatment.

It has been investigated that several mechanisms are involved in the magnetic inductive heating characteristic of the ferromagnetic materials. While Brownian movement and Neel relaxation mainly account for the inductive heating of the nano- or micro-scaled metal materials, Eddy current loss induced by the AMF would provide a continuous heat source through resistive heating of the milliscaled materials, such as 
the esophageal stents applied in this study (15). Briefly, the magnetic field induces current to flow in the stent, the resistance of the stent impedes the current flow, therefore producing heat. Though higher frequency and intensity leads to higher stent temperatures, heating of the surrounding tissue by electromagnetic effects is also more pronounced with high AMF parameters. Therefore, in order to exclude the surrounding tissue from heating and to deliver energy selectively into the stent, there is an upper limit of frequency that should not be exceeded. It was reported by Floren et al that $200 \mathrm{kHz}$ would be the favorable frequency to minimize warming of muscle tissue, skin, and fat for human applications (16). With a working frequency of around $110 \mathrm{kHz}$, our applicator is with full compliance to the safety regulations demanded in clinical applications.

A very important issue associated with magnetic hyperthermia is the contribution of AMF exposure on the hyperthermic cytotoxicity. Currently, the field frequency of AMF for magnetic hyperthermia belongs to the intermediate frequency range. Field effects of frequencies up to $1 \mathrm{kHz}$ and above $1 \mathrm{MHz}$ are well known, however, the intermediate frequency range lacks intensive research and only little attention has been paid so far. Our investigation proved that AMF exposure has a small inhibitory effect on the proliferation of ECA-109 cells, and does not have an effect on cell apoptosis and necrosis. A detailed study should be carried out for a better understanding on the biological effect of AMF exposure and such study is now underway.

For cancer treatment, the effectiveness of hyperthermia is related to the temperature achieved during the treatment. In order to sacrifice cancer cells without injury to normal tissues, the choice of temperature is important in hyperthermia therapy. Besides, the total treatment time is another key factor involved with thermal dose when prescribing hyperthermia treatment (17). The current study demonstrated that a mild temperature of $43^{\circ} \mathrm{C}$ has very little effect on the viability and necrosis of the ECA-109 cell in the time range of 5-30 min. It thus can be concluded that in the clinical treatment of esophageal cancer by hyperthermia, a higher temperature should be adopted. Except for esophageal cancer cells, it has been shown by other researchers that $43^{\circ} \mathrm{C}$ is not an effective hyperthermic temperature. For instance, Ito et al (18) found that $43^{\circ} \mathrm{C}$ can even facilitate the tumor growth of malignant melanoma in C57BL/6 mice.

It is normally believed that for certain hyperthermic temperatures, the better therapeutic effect can be achieved by prolonging the treatment time. However, for the case of esophageal cancer cells, the asymptote-shaped cell viability curve strongly implies that during the clinical hyperthermia treatment for esophageal cancer, prolonging the treatment time may not necessarily improve the therapeutic effect. The thermal dose of $48^{\circ} \mathrm{C}$ hyperthermic temperature with 20-30 min treatment time may incur similar inhibitory effects on cell viability as a higher temperature of $53^{\circ} \mathrm{C}$ for the same treatment time. We thus can deduce from the in vitro investigation that the hyperthermic temperature of $48^{\circ} \mathrm{C}$ with 20-30 min exposure times may be the optimal thermal dose for in vivo testing.

Taken together, MSH can lead to an inhibitory effect on the viability of ECA-109 cells with optimal thermal dose of 20-30 min exposure time under $48^{\circ} \mathrm{C}$ heating treatment.
Exposure of ECA-109 cells under AMF with a field intensity scope of $50-110 \mathrm{kA} / \mathrm{m}$ has negligible effect on cell viability, cell necrosis and apoptosis. The clinical esophageal stent possesses excellent inductive heating properties which can greatly facilitate the clinical application of MSH for esophageal cancer treatment.

\section{Acknowledgements}

This study was supported by the National Natural Science Foundation of China (no. 81041040 to L.-Y. L. and no. 30973439 to L.-Y. Z.) and the China Postdoctoral Science Foundation (no. 20080430045 to L.-Y. Z.).

\section{References}

1. Wang ZG, Fukazawa T, Nishikawa T, et al: RAD001 offers a therapeutic intervention through inhibition of mTOR as a potential strategy for esophageal cancer. Oncol Rep 23: 1167-1172, 2010.

2. Gockel I, Moehler M, Frerichs K, et al: Co-expression of receptor tyrosine kinases in esophageal adenocarcinoma and squamous cell cancer. Oncol Rep 20: 845-850, 2008.

3. Candeo A and Dughiero F: Numerical FEM models for the planning of magnetic induction hyperthermia treatments with nanoparticles. IEEE Trans Magn 45: 1658-1661, 2009.

4. Johannsen M, Thiesen B, Jordan A, et al: Magnetic fluid hyperthermia (MFH) reduces prostate cancer growth in the orthotopic Dunning R3327 rat model. Prostate 64: 283-292, 2005.

5. Deger S, Taymoorian K, Boehmer D, et al: Thermoradiotherapy using interstitial self-regulating thermoseeds: an intermediate analysis of a phase II trial. Eur Urol 45: 574-580, 2004.

6. Deger S, Boehmer D, Türk I, Roigas J, Budach V, Loening SA: Interstitial hyperthermia using self-regulating thermoseeds combined with conformal radiation therapy. Eur Urol 42: 147-153, 2002.

7. Jordan A, Scholz R, Maier-Hauff K, et al: The effect of thermotherapy using magnetic nanoparticles on rat malignant glioma. J Neurooncol 78: 7-14, 2006.

8. Cherukuri P, Glazer ES and Curley SA: Targeted hyperthermia using metal nanoparticles. Adv Drug Deliv Rev 62: 339-345, 2010.

9. Ito A and Kobayashi T: Intracelluar hyperthermia using magnetic nanoparticles: a novel method for hyperthermia clinical applications. Thermal Med 24: 113-129, 2008.

10. Yambe T, Hori Y, Shiraishi Y, Sekine K and Miyata G: Drinking stent with therapeutic hyperthermia effect for esophageal cancer. Int J Artif Organs 29: 548, 2006.

11. Akiyama S, Kawasaki S, Kodera Y, et al: A new method of thermo-chemotherapy using a stent for patients with esophageal cancer. Surg Today 36: 19-24, 2006.

12. Zhou JM, Li N, Xia QS, et al: Hyperthermia by a nitinol stent in an alternating magnetic field: safety and feasibility in rabbit esophageal cancer. Prog Nat Sci 19: 1713-1719, 2009.

13. Wan XA, Sun GP, Wang H, Xu SP, Wang ZG and Liu SH: Synergistic effect of paeonol and cisplatin on oesophageal cancer cell lines. Dig Liver Dis 40: 531-539, 2008.

14. Pallepati P and Averill-Bates DA: Mild thermotolerance induced at 40 degrees $C$ protects HeLa cells against activation of death receptor-mediated apoptosis by hydrogen peroxide. Free Radic Biol Med 50: 667-679, 2011.

15. Wang XF, Tang JT and Shi LQ: Induction heating of magnetic fluids for hyperthermia treatment. IEEE Trans Magn 46: 1043-1051, 2010.

16. Floren MG, Günther RW and Schmitz-Rode T: Noninvasive inductive stent heating: alternative approach to prevent instent restenosis. Invest Radiol 39: 264-270, 2004.

17. Gerner EW: Thermal dose and time-temperature factors for biological responses to heat shock. Int J Hyperthermia 3: 319-327, 1987.

18. Sato A, Tamura Y, Sato N, et al: Melanoma-targeted chemo-thermo-immuno (CTI)-therapy using N-propionyl-4-Scysteaminylphenol-magnetite nanoparticles elicits CTL response via heat shock protein-peptide complex release. Cancer Sci 101: 1939-1946, 2010. 